\title{
EDUCACIÓN A DISTANCIA HOY: EN BUSCA DE LA COMUNICACIÓN REAL
}

(DISTANCE EDUCATION TODAY: IN SEARCH OF THE REAL COMMUNICATION)

\author{
Mónica Isabel Perazzo Puncel \\ Universidad Nacional de Lanús (Argentina)
}

\section{RESUMEN}

El presente artículo se propone aportar algunas reflexiones a partir del análisis de la comunicación didáctica que se ha realizado en un universo de programas de educación a distancia que se desarrollan total o parcialmente en entornos virtuales en Argentina. Además de explorar evidencias sobre las características y los sentidos que asume la comunicación educativa tanto en el diseño como en el desarrollo y gestión de propuestas concretas, este trabajo identifica algunos flancos aún oscuros o difusos de los circuitos de interacción que entablan profesores y estudiantes durante los trayectos a distancia y que merecen atención para redireccionar los mismos hacia mejores formas de enseñar y aprender.

Palabras clave: educación a distancia, comunicación educativa, interacción en línea, entornos virtuales de aprendizaje.

\begin{abstract}
This article aims to provide some considerations from the analysis of didactic communication made in a world of distance education programs that are developed in whole or in part in virtual environments in Argentina. Moreover to exploring the characteristics and evidence of the senses that takes the educational communication in the design and development of specific proposals, this paper identifies some still obscure and fuzzy aspects of the channels of interaction established between teachers and students during the distance routes and for attention to redirect them towards better ways to teach and to learn.
\end{abstract}

Keywords: distance education, educational communication, online interaction, virtual learning environments.

Este artículo, cuyo título evoca una célebre obra de la literatura universal: "En busca del tiempo perdido” de Marcel Proust, se propone analizar y reflexionar sobre 
el lugar real de la comunicación en la educación a distancia de nuestro tiempo, esto es, su presencia en el diseño y en el desarrollo efectivo de programas que se ofrecen por entornos virtuales o a través de instancias combinadas (presenciales y virtuales). En el contexto de este relato el adjetivo real no se opone a lo virtual sino a lo potencial y por ello, se trata de indagar qué tipo de comunicación se instala desde el diseño y desde la gestión concreta del proceso educativo en el escenario actual donde las tecnologías de la información y de la comunicación (TIC) ofrecen múltiples alternativas de relación y nuevas formas de enseñar y aprender.

Además de explorar las características y los sentidos que asume la comunicación en propuestas a distancia, este trabajo pretende analizar su dimensión más significativa en términos de lograr intercambios y diálogos entre los actores, y reflexionar sobre algunas evidencias que nos muestran casos singulares que se han desarrollado en los últimos cinco años en las distintas jurisdicciones que conforman la República Argentina. Esta exploración posibilita identificar algunos flancos aún oscuros o difusos de los circuitos de interacción que entablan profesores y estudiantes durante los trayectos específicos de educación a distancia, en especial dentro de los campus virtuales, con el propósito de orientar o redireccionar las propuestas hacia otros horizontes y contribuir al mejoramiento de la calidad de los estudios.

\section{TERRITORIO SEMÁNTICO PARA COMPARTIR}

En primer lugar se hace imprescindible marcar territorio semántico sobre qué entendemos por comunicación, una palabra polisémica que en el campo de la educación adquiere rasgos sustantivos. El lenguaje coloquial y hasta el debate académico sobre la comunicación se perfilaron en forma sesgada durante años reduciéndola a una cuestión de mera trasmisión de mensajes o de información mediada por tecnologías masivas donde el espectáculo y la fascinación siguen siendo rasgos distintivos y donde los medios se consideran como únicos objetos legítimos para el estudio de la comunicación. Esta perspectiva limitada omite, soslaya o desconoce que el núcleo sustantivo de la comunicación reside en la relación interpersonal, en la interacción, en el encuentro de subjetividades, en el diálogo con lenguajes verbales y no verbales y en la influencia mutua entre dos o más personas. Así desde una vertiente más profunda, cuando se habla de comunicación nos remitimos al mundo de las relaciones y de los vínculos entre sujetos, de los conflictos, encuentros, desencuentros, malentendidos, de los diálogos y monólogos, de los ruidos y de las intolerancias, de las palabras y de los 
silencios, de los tonos de voz, de los ademanes y gestos universales y singulares que se configuran a través de la vida cotidiana.

$\mathrm{Al}$ recuperar el origen del término comunicación se constata que la raíz latina "communis" -presente también en las palabras comunidad y comunión- forma parte de "communicare" que significa poner en común y compartir algo, lo cual confirma que existe comunicación cuando dos o más personas ponen en común ideas, sentimientos y emociones, a través del lenguaje verbal y no verbal. Se trata de un proceso de ida y vuelta de mensajes donde los sujetos -aplicando el neologismo de Jean Cloutier- son "emirecs" porque simultáneamente son emisores y receptores de significados en un contexto singular, situación que lleva a Jesús Martín-Barbero (1996, p. 11) a sostener que "La comunicación es diálogo en la trama cultural".

Entre los especialistas que abonan esta perspectiva de la comunicación como interacción, está Tim O’Sullivan (1997) quien expresa que la interacción puede ser entendida como "el intercambio y la negociación del sentido entre dos o más participantes situados en contextos sociales” resaltando que en esa interacción social hay un rasgo de reciprocidad entre quienes utilizan y construyen códigos y reglas. Habermas (1986, p. 68-69) también postula la relevancia de la interacción al sostener "Por acción comunicativa entiendo una interacción simbólicamente mediada. Se orienta de acuerdo con normas intersubjetivamente vigentes que definen expectativas recíprocas de comportamiento y que tienen que ser entendidas y reconocidas, por lo menos por dos sujetos agentes".

Con sentido metafórico Jesús Galindo (2004) visualiza a la interacción como el "corazón" de la comunicología dando cuenta de la mutua afectación que tiene lugar entre sujetos en la vida amorosa, familiar, vecinal, laboral, comunitaria y social.

Situados en el ámbito de la educación, la comunicación se configura como un acto esencial, imprescindible y sustantivo para sostener la relación docentealumno y para el desarrollo de una propuesta formativa siendo en el interior de las aulas dedicadas a la enseñanza presencial un proceso consustancial, continuo y transparente mientras que en la modalidad a distancia adquiere atributos especiales algunos de los cuales se van a dilucidar en este trabajo.

Sobre la base de las referencias sociológicas de Berger y Luckmann (1972) se puede afirmar que la relación cara a cara -entre docente y alumnos- se caracteriza por una presencia inmediata, continua y real de la expresividad que involucra todos los lenguajes humanos: los verbales que se traducen en códigos determinados, 
arbitrarios y convencionales, y los no verbales que se reflejan en la mirada, la escucha, la voz, la gestualidad expresiva, el contacto y la actitud postural. Desde ese punto de vista, nuestro rostro, nuestra voz y nuestro cuerpo son vertientes naturales y permanentes de expresividad a la vez que instrumentos que comunican, tal como lo sostiene Roland Barthes (1993, p. 148): "Puedo hacerlo todo con mi lenguaje, pero no con mi cuerpo. Lo que oculto mediante mi lenguaje lo dice mi cuerpo. Puedo modelar mi mensaje a mi gusto, pero no mi voz. En mi voz, diga lo que diga, el otro reconocerá que 'tengo algo'"

Retomando el contexto de una relación cara a cara, Berger y Luckmann (1972. p. 46-48) afirman que ahí se produce "un intercambio continuo entre mi expresividad y la del otro", una "continua reciprocidad de actos expresivos" y una "abundancia de señales, síntomas de subjetividad que yo puedo identificar en el otro; hay variedad en el intercambio de significados". Los docentes que habitan el ámbito educativo sienten y perciben al aula como ese espacio único y específico plagado de intercambios explícitos o subterráneos, propósitos pretendidos, buscados y no buscados, emergentes cotidianos que hablan de intereses y expectativas de profesores y estudiantes, conductas y mensajes verbales y no verbales donde se entremezclan y se amalgaman sensaciones, afectos, emociones, subjetividades e ideas.

Frente al derroche de vivacidad y expresividad que caracteriza a la presencialidad, surge la pregunta: ¿Se pierden, se ocultan, se sustituyen esos actos expresivos no verbales, esas señales y síntomas de subjetividad en la educación a distancia? ¿Los estudiantes tienen pocas y acotadas oportunidades de decir su palabra? ¿Pierden su protagonismo como interlocutores y sujetos del diálogo? ¿La retroalimentación inmediata y continua es casi inexistente o se reduce sensiblemente? ¿Qué efectos y consecuencias formativas surgen ante esas carencias y limitaciones?

Desde la vertiente de la Escuela de Palo Alto, Watzlawick y otros especialistas (1991, p.50) sostienen que es imposible no comunicarse y agregan: "Si se acepta que toda conducta en una situación de interacción tiene un valor de mensaje, es decir, es comunicación, se deduce que por mucho que uno lo intente, no puede dejar de comunicar". A partir de ese encuadre surgen inevitablemente ciertas preguntas: Ese principio que para los mencionados autores es un axioma ¿̇se convierte en hipótesis en la educación a distancia? Ese postulado que abunda en certezas en el marco del aula convencional ¿cómo se proyecta e interpreta en la educación a distancia? 
Dentro del repertorio de interacciones y de prácticas posibles en la educación, Burbules(1999,p.32)entiendealdiálogocomounarelación comunicativapedagógica que exige un compromiso emocional y cognitivo de los sujetos participantes y que entraña múltiples implicancias para la vida personal y social. Agrega: "El diálogo no es como otras formas de comunicación (charlar, argumentar, negociar, etc.). El diálogo es una actividad dirigida al descubrimiento y a una comprensión nueva, que mejora el conocimiento, la inteligencia o la sensibilidad de los que toman parte en él."

Desde la pedagogía de Paulo Freire (1976) la educación es una práctica donde se pone en juego la intersubjetividad a través del diálogo y la palabra, mientras que otros enfoques socioculturales refieren a la comunicación no sólo como la base de la interacción social sino como el componente necesario del proceso cognitivo: cuando el sujeto logra expresar una idea o un concepto de modo que otros puedan comprenderlo, él mismo aprende, comprende y se apropia de su sentido (Jorba, Gómez A. y Prat, 2000).

\section{PERSPECTIVAS TEÓRICAS SOBRE LA COMUNICACIÓN DIDÁCTICA EN PROPUESTAS A DISTANCIA}

La educación a distancia, por definición, supone un proceso de comunicación en gran parte o totalmente mediado, donde la interacción profesor y estudiantes se apoya y tiene lugar a través de distintos medios culturales y tecnológicos (Moore, 1975; Kaye y Rumble, 1979; Holmberg, 1985). La clásica tríada docente-alumnocontenido asume rasgos diferentes a la educación presencial ya que se despliega a través de diferentes recursos y soportes tecnológicos que son objeto de un tratamiento didáctico específico. Así la mediación didáctica a distancia (Gutiérrez y Prieto Castillo, 1999) impone condiciones singulares para organizar y propiciar los aprendizajes y establecer el tipo de relaciones entre profesor y alumnos, y se plasma no sólo en los materiales de estudio -considerados como objetos culturales y tecnológicos que posibilitan construir sentidos- sino también en los dispositivos de intervención y apoyo permanentes y continuos que realizan los profesores.

Teniendo en cuenta que nuestro foco de interés está en el análisis de la comunicación real entendida como interacción y diálogo, es oportuno hacer un breve recorrido sociohistórico sobre las formas y las tecnologías que sustentaron la comunicación de la educación a distancia de ayer y hoy. Este recorte del objeto de estudio no debe eximirnos de considerar una perspectiva más amplia donde 
el desarrollo de esta modalidad configura un proceso histórico-social complejo, heterogéneo y no lineal donde tienen lugar distintas situaciones que implican avances, retrocesos, rupturas y discontinuidades en torno a su sentido y valor social. Se trata de una trama tejida por distintos sujetos y componentes donde se enlazan aspectos políticos, culturales, sociales, didácticos y tecnológicos.

Desde esa impronta el itinerario o recorrido que se plantea pretende graficar a grandes rasgos un proceso sociohistórico complejo y multidimensional donde han convivido y donde se han fusionado distintas expresiones, prácticas, ritos y subjetividades sobre el valor de la educación a distancia, y dentro de él se busca explorar algunas cuestiones sobre la comunicación potencial y la real en diferentes momentos de su desarrollo.

Sobre la base de lo expuesto por distintos autores (Niper, 1989; Lauzon y Moore, 1989; Taylor, 2001) que han definido etapas o generaciones de educación a distancia que se han configurado en el tiempo, este trabajo aborda cuatro generaciones colocando el énfasis en el análisis de la comunicación didáctica, en la interacción entre docente y alumnos, y alumnos entre sí, con la idea de reconocer algunas de sus peculiaridades, limitaciones y debilidades.

En el estadio inicial, la educación a distancia era por correspondencia con uso de material impreso como medio principal, con eventual apoyo de otras mediaciones: tutorías por correo postal, algunas consultas por teléfono (objeto de difícil acceso en ese entonces), y en algunos casos, reuniones presenciales con la coordinación de un profesor-tutor o facilitador.

Los resultados de los desarrollos de esta etapa con esos medios disponibles para la comunicación, podrían resumirse en que eran casi inexistentes o bajas las posibilidades de interacción directa, simultánea e inmediata entre docente y alumnos, y estudiantes entre sí. Había predominio de esquemas unidireccionales de la comunicación: emisión de mensajes sin registro de recepción de esos mensajes, de sus influencias y efectos. La retroalimentación se tornaba difícil, lenta y por ello, de baja incidencia para ajustar y orientar oportunamente el proceso de aprendizaje. La interacción bidireccional se podía concretar en forma sincrónica con el teléfono y de manera asincrónica con el correo postal. En suma, las posibilidades comunicativas reales, en el sentido de intercambios e interacciones socio-cognitivas, eran escasas, diferidas, discontinuas, con mínima presencia de señales analógicas y expresivas que dieran lugar al encuentro de emociones, expectativas y motivaciones de los actores. 
Más allá de esos rasgos definidos, es importante señalar que en la República Argentina como en otros países de América Latina, hubo significativas experiencias de educación popular y no formal a distancia para adultos que se basaron en el trabajo periódico con grupos para promover el desarrollo comunitario y regional, como Incupo de Argentina, Radio Sutatenza, Asociación Latinoamericana de Educación Radiofónica, entre otros (La Belle, 1980).

En una segunda generación o estadio, los medios instrumentales de la educación a distancia se incrementaron. A los medios impresos se sumaron la radio, la televisión, el audio-casete y el video (Casas Armengol, 1987). Los apoyos tutoriales mantenían su lugar a través de mensajes que se podían realizar por correo postal y por teléfono, un poco más popular para ese entonces, mientras que algunas experiencias incluían encuentros periódicos presenciales para alentar y propiciar intercambios diversos, trabajos prácticos y evaluaciones.

Los resultados de esta etapa se pueden traducir en que los medios audiovisuales aportaban mayor variedad y riqueza de lenguajes -verbales y no verbales-, más estímulos visuales y nuevas fuentes de representación de conocimientos. No obstante, en los programas subsistían carencias en la comunicación por las bajas posibilidades de interacción directa, simultánea e inmediata entre docente y alumnos, y de pares entre sí (Kaplún, 1983).

La comunicación a través de esos medios, seguía siendo básicamente diferida, discontinua, con poca presencia de códigos analógicos y expresivos de los actores, y la retroalimentación inmediata para apoyar y orientar de manera oportuna y efectiva los aprendizajes, resultaba precaria y limitada con las implicancias que ello supone para la motivación y desempeño del alumno.

En el tercer momento o generación se destacó la aplicación de la computadora y la informática que como nuevas tecnologías aportaron ventajas en el almacenamiento, transporte y rapidez de la información (Casas Armengol, 1987). Los resultados manifiestos destacan la existencia de un nuevo, flexible y caudaloso soporte para la información y nuevos saberes asociados a su uso dando lugar al concepto de "residuo cognitivo" que Salomon y otros (1992) definen como las habilidades y estrategias de pensamiento que se producen como efecto de la relación sujeto y tecnología que le permiten reorganizar y aumentar su rendimiento incluso cuando se encuentra apartado de la tecnología en cuestión. En cuanto a la dimensión comunicativa propiamente dicha, en este período persistían las bajas posibilidades de interacción entre los sujetos que enseñan y aprenden, excepto en las experiencias 
que imponían reuniones y encuentros periódicos o en aquellas donde el teléfono y el fax se tornaban recursos accesibles y de bajo costo para los grupos destinatarios (Perazzo, 2002; Barberá, 2008).

En la década de los 90 surgió el cuarto momento o generación actual con el auge de las redes telemáticas sustentadas en el avanzado desarrollo de la informática y de las telecomunicaciones cuyo mayor exponente es internet con sus múltiples herramientas y servicios de información y de comunicación (Lauzon y Moore, 1989; Pérez Tornero, 2000) que ganan espacio en la comunidad educativa. Se diseñan y producen materiales didácticos hipertextuales e hipermediales que ofrecen diversidad de fuentes de información y de lenguajes que superan las limitaciones de los textos lineales y secuenciales en soporte impreso y la comunicación se hace posible con herramientas variadas y versátiles como correo electrónico, foros, chats, videoconferencias, blogs o bitácoras, wikis, redes sociales, entre otras.

Las plataformas de teleformación, los entornos o campus virtuales son los modelos y epicentros que posibilitan y recrean ámbitos de enseñanza y de aprendizaje a la manera de las aulas convencionales con fuertes implicancias para los modos de conocer y aprender (Duart y Sangrá, 2000; Pérez Tornero, 2000), además de ofrecer un gran potencial para generar comunicaciones simultáneas, directas y rápidas, bi y multidireccionales, sincrónicas y asincrónicas entre los actores, más allá de la distancia geográfica y de las situaciones personales, sociales y laborales que definen sus condiciones de vida. No obstante para que ese potencial sea efectivo se torna imprescindible el tratamiento didáctico a cargo de pedagogos y profesores, tal como lo sostiene Barberá (2008:26): “...En esta modalidad educativa es la calidad del proceso de enseñanza la que marca la diferencia entre un aprendizaje más profundo y un aprendizaje más superficial (Saljö, 1979)” Y agrega: "En este marco, se entiende que la construcción del conocimiento humano se produce debido a la interrelación entre tres elementos instruccionales: el alumno en cuanto aprendiz, los contenidos que son objeto de enseñanza y aprendizaje, y el profesor, que colabora en la construcción de significados y en la atribución de sentido al contenido compartido" (Barberá, 2008, p. 30)

En ese escenario caracterizado por las posibilidades comunicativas, muchas de las interacciones siguen desprovistas de los marcadores del lenguaje gestual y no verbal, aspecto que abordan García Carrasco y Álvarez Cadavid (2008) cuando argumentan: "La configuración de la interacción entre individuos en ambientes virtuales está compuesta por una trama de mensajes, en su mayoría textuales; al no estar presentes en Internet las claves de la corporeidad física y del contexto, 
las da el texto, que al igual que en la literatura es el portador de los atributos de identidad".

Otra vertiente potencial de los entornos virtuales es que abren perspectivas para promover aprendizajes de tipo colaborativo y para conformar redes sociales en torno a intereses y necesidades comunes de personas y grupos. Desde esa línea, Ardizzone y Rivoltella (2004, p. 118) expresan que el estudiante de cursos en línea tiene posibilidades de mayor implicación cuando la plataforma a través de las zonas de interacción y de edición, lo autoriza a organizar elementos del ambiente colectivo. Los autores entienden que así “...se abren nuevos espacios para actividades de colaboración: si bien éstas son decididas normalmente por el profesorado y a menudo están fijadas ya en el modelo del curso, se trata de actividades que valoran la aportación del alumno, emancipándolo de la posición de receptor final de la información en la que muchas veces corre riesgo de quedar confinado".

La gestión colectiva del conocimiento y la elaboración compartida de proyectos y recursos educativos hoy son posibles gracias a las herramientas de la web 2.0, también llamada red participativa, en la que los blogs o bitácoras, las wikis, los agregadores de noticias (RSS), la clasificación y distribución de contenidos y las redes sociales se multiplican y se propagan día a día en el mundo académico (Fumero, Roca, y Sáez Vacas, 2007).

Sin embargo, más allá de las potencialidades y virtudes asignadas a las TIC, que los actores mantengan interacciones e intercambios socio-cognitivos en el contexto educativo sigue siendo un desafío y una hipótesis de trabajo, tal como lo expone Barberá (2008, p. 42): "En suma, las nuevas tecnologías proporcionan a la educación contextos cualitativamente diferentes que permiten cuestionar el carácter privado que se ha atribuido tradicionalmente al proceso de aprendizaje a distancia, así como también replantear los modelos centrados en la transmisión de información estandarizada a un elevado número de alumnos, transmisión con pocas posibilidades de interacción y que conlleva una asimilación pasiva y poco crítica de la información".

\section{UN AMPLIO OBSERVATORIO DE EDUCACIÓN A DISTANCIA}

Las referencias y reflexiones que se aportan en este trabajo se han construido a partir del análisis de numerosos proyectos a distancia de educación secundaria para 
jóvenes y adultos y de educación superior que han adoptado total o parcialmente plataformas y entornos virtuales para sus programas, y que fueron presentados para su evaluación en la Comisión Federal de Registro y Evaluación Permanente de las Ofertas de Educación a Distancia, de Argentina, que funciona desde el año 2003. La magnitud del campo observado puede reconocerse en la cantidad de propuestas analizadas en el período 2003-2008 que alcanzó las 980 y que provenían de todas las jurisdicciones del país. De esa cifra, un porcentaje cercano al $75 \%$ fue evaluado en dos y hasta tres ocasiones, en distintas convocatorias, en virtud de que las instituciones debían redefinir y mejorar determinadas dimensiones de sus propuestas pedagógicas.

El análisis de las experiencias, que implica una lectura orientada por determinado marco teórico (Kaplún, 1983; Holmberg, 1985; Borrás, 1997; Perazzo, 2002) que sustenta el equipo de evaluadores y que supone un acto de interrogación que se proyecta sobre el universo de casos estudiados, ha permitido formular estas aproximaciones y reflexiones que son personales aunque devienen de un trabajo colectivo realizado en el período 2003-2008.

A partir del observatorio disponible y del amplio mapa de casos donde el foco se instaló en las comunicaciones didácticas e interacciones entre profesores y estudiantes de entornos virtuales y de programas de "nivel compartido" (Abbey, 2000) que combinan trayectos presenciales y virtuales, se analizaron las propuestas de cada organización educativa de acuerdo con un protocolo previamente establecido que incluía la presentación de documentos curriculares y de gestión académica, además de materiales pedagógico-didácticos.

\section{ANÁLISIS DE CASOS}

Para realizar el análisis de la calidad de las interacciones socio-cognitivas entre profesores y estudiantes, y estudiantes entre sí, en carreras de nivel medio y superior, se definieron las siguientes dimensiones y elementos que, en forma conjunta e interrelacionada, dan identidad a cada propuesta educativa virtual:

- Diseño pedagógico-didáctico del proyecto

- Elaboración de los materiales de estudio y de las propuestas incluidas en el entorno virtual: 
- $\quad$ Grado de explicitación y especificación de los objetivos, actividades y consignas de trabajo para el intercambio y la elaboración conjunta y compartida de conocimientos.

- Espacios y tutorías que involucren interacciones socio-cognitivas entre alumnos y profesores: obligatorias y opcionales, grupales y colectivas.

- Coherencia o grado de concordancia entre el diseño pedagógico-didáctico, la propuesta plasmada en el entorno y el desarrollo efectivo de las interacciones socio-cognitivas.

Las fuentes consultadas en cada caso fueron: normativa, proyecto pedagógico institucional, diseño curricular, propuesta de enseñanza, materiales de estudio, modelos de evaluación del aprendizaje, arquitectura y funcionamiento del campus o plataforma, y dispositivo para el monitoreo y evaluación del proyecto. Las técnicas de recolección de datos fueron básicamente el análisis documental y el ingreso a la plataforma para indagar y verificar el desarrollo y gestión académica de los cursos.

A continuación se destacan algunas de las conclusiones esbozadas a partir de la información recogida en la indagación del campo:

\section{DISEÑO PEDAGÓGICO-DIDÁCTICO DEL PROYECTO}

Esta dimensión se refiere al proceso de selección y formulación de un conjunto articulado de contenidos, estrategias metodológicas y herramientas tecnológicas que, sustentado en marcos teóricos específicos, impliquen diferentes tipos de interacciones entre profesores y estudiantes, y estudiantes entre sí, orientados al logro de los objetivos de aprendizaje en un contexto determinado. El sentido que adquiere el diseño es asumido por Guardia (2000, p. 172) cuando señala: "La necesidad evidente, pues, de un máximo aprovechamiento pedagógico de la tecnología y de las herramientas multimedia hace emerger el concepto de diseño formativo como proceso imprescindible que define y concreta cómo tienen que ser todos los elementos que configuran una acción formativa".

En los casos abordados se observa que prevalece el diseño acotado al empleo de las plataformas como reservorios para la publicación lineal de los programas, clases y desarrollos teóricos, ejercicios y trabajos prácticos, bibliografía y calendario, siendo usual que la versión impresa de los materiales de estudio se digitalice, se 
replique y se reproduzca en el campus virtual sin ningún valor agregado ya que se omiten espacios activos para el intercambio cognitivo. Ello da cuenta de una escasa incidencia pedagógica-comunicativa de las herramientas tecnológicas que están disponibles en casi todas las plataformas y un uso precario, limitado y restringido de las variadas formas que existen para recrear, generar y mantener los diálogos y debates que promuevan la socialización académica y la socio-construcción compartida de los conocimientos.

En el caso de carreras de educación superior cuyos perfiles profesionales requieren el cumplimiento de observaciones, prácticas y pasantías, es común que los diseños pedagógicos restrinjan su desarrollo al estudio de casos escritos y a la aplicación de simulaciones, sin propiciar la inserción del alumno en organizaciones y contextos de trabajo vinculados con los ámbitos de desempeño profesional. De esa manera los futuros egresados no disponen de espacios reales y situados para expresar y contrastar la integración y articulación de los saberes teórico-prácticos con el seguimiento y apoyo de profesores.

En relación con el grado de aprovechamiento de las herramientas para la comunicación Marcelo García y Perera Rodríguez (2007, p. 423) al investigar sobre las interacciones en ambientes virtuales señalan que: "Creemos que el medio sí posibilita el desarrollo de una formación donde la comunicación e interacción entre alumnos y profesores se produzca de manera más natural y horizontal."

Desde esa línea, el acceso al observatorio dio cuenta de que sólo un ínfimo porcentaje de los casos aprovecha el potencial comunicacional de los recursos para crear y promover ambientes de intercambio en foros, blogs y chats orientados a la resolución de problemas, al debate grupal sobre ejes temáticos y a la elaboración de proyectos colaborativos. Esta subutilización de las posibilidades comunicativas sincrónicas y asincrónicas de los entornos se traduce en la ausencia de fecundas comunidades discursivas donde los actores puedan compartir ideas, miradas, prácticas, experiencias y reflexiones (Borrás, 1997; Perazzo, 2002; Barberá, 2008).

ELABORACIÓN DE LOS MATERIALES DE ESTUDIO Y DE LAS PROPUESTAS INCLUIDAS EN EL ENTORNO VIRTUAL

Grado de explicitación y especificación de los objetivos, actividades y consignas de trabajo para el intercambio y la elaboración conjunta y compartida de conocimientos 
En los casos en que se han incluido herramientas virtuales para la comunicación, su uso es anodino, con propósitos difusos o vagamente establecidos, con temas y debates en foros que se plantean sin moderación alguna ni cierre provisorio, actividades académicas opcionales que no tienen seguimiento ni evaluación, chats sin pautas claras para el intercambio académico o metodológico, información distribuida en distintas secciones de la plataforma con criterios imprecisos y calendarios desactualizados que ponen de manifiesto la discontinuidad de actividades.

Surge del análisis que esos espacios virtuales, además de no plantearse con criterios académicos claros y precisos que fijen objetivos y períodos de discusión, responsabilidades en la moderación y sistematización de los aportes individuales, dejan librado al estudiante su participación ya que no se definen como exigencias académicas, lo cual muestra las escasas oportunidades que ofrece la propuesta para las interacciones socio-cognitivas.

Desde una perspectiva del aprendizaje que privilegia la co-construcción de significados y saberes, investigadores como Gunawardena, Lowe y Anderson (1997) han analizado y definido diferentes niveles de construcción social o compartida del conocimiento a partir de las interacciones realizadas en ambientes virtuales. El modelo que esos autores han formulado comprende cinco fases o niveles posibles de intercambios, con un creciente grado de complejidad, en el cual el nivel más simple está dado por los hechos comunicativos a través de los cuales los estudiantes comparten mera información, y el nivel más complejo involucra tanto la aplicación de los nuevos significados que han llegado a construir los integrantes de un grupo como la expresión de logros metacognitivos como resultado del proceso de aprendizaje. Así en un primer momento o fase I los estudiantes comparten y comparan información sobre determinado tema o problema, clarifican significados de términos, enuncian opiniones, ejemplos, preguntan y cuestionan elementos de los enunciados.

Para Gunawardena y otros, la fase II se caracteriza por el descubrimiento y exploración de la disonancia o inconsistencia de ideas, conceptos o enunciados, donde los alumnos identifican y establecen desacuerdos y re-establecen argumentos y referencias, mientras que el nivel III avanza en la negociación de significados para llegar a la co-construcción del conocimiento, con identificación de acuerdos, de términos conflictivos e integración de analogías y metáforas. Una posición más elevada reside en la fase IV cuando los participantes llegan a la prueba y modificación de la síntesis conceptual o co-construcción del conocimiento, mientras 
que el nivel V, el máximo en esta escala, se alcanza cuando los estudiantes resumen los enunciados acordados y los nuevos significados construidos y probados en diferentes situaciones y, además, expresan las metacogniciones que han logrado durante el aprendizaje compartido.

Este modelo, y el propuesto por Garrison y Anderson (2005) que formula tres grandes tipos de presencias: cognitiva, social y docente, constituyen referencias significativas para diseñar, organizar y gestionar los circuitos de comunicación didáctica en los entornos virtuales de aprendizaje de modo tal que los diálogos e intercambios -con la orientación y mediación de los profesores- fluyan hacia objetivos y competencias socio-cognitivas de creciente complejidad.

Otro aspecto a considerar en este apartado es el referido al análisis de los instrumentos de evaluaciones parciales y finales del aprendizaje que pongan en juego el logro de construcciones socio-cognitivas. Al respecto, los casos analizados dan cuenta de un predominio de instrumentos escritos basados en el diseño estructurado -pruebas "objetivas"-, lo cual supone, por un lado, escasas oportunidades para que el estudiante pueda analizar, argumentar y elaborar trabajos que reflejen cabalmente las construcciones cognitivas logradas y, por otro, que determinados objetivos de aprendizaje queden sin posibilidades de verificación y expresión genuina y que no se puedan identificar parámetros para la revisión de la propuesta académica, afectando así la formación de competencias que ella pretende.

\section{Espacios y tutorías que involucren interacciones socio-cognitivas entre alumnos y profesores: obligatorias y opcionales, grupales $y$ colectivas}

Otra vertiente que permite dar cuenta de la comunicación didáctica que plantea una propuesta a distancia es la que proviene de un análisis basado en una "estrategia deconstructiva" orientada a deconstruir y develar los puentes, intersticios y atajos del circuito comunicativo. En tal sentido, desde el observatorio se han analizado diferentes indicadores como: los espacios previstos -obligatorios y opcionales- para generar las interacciones, los sujetos participantes, las exigencias académicas, el tipo de actividades propuestas, las formas e instancias de apoyo, seguimiento y ajuste de tareas, los instrumentos y periodicidad de los diálogos, los roles y los desempeños asignados a cada participante, entre otros. Todos ellos aportan elementos, señales e indicios que permiten reconstruir la estructura y organización del dispositivo y flujo de comunicación que las organizaciones han 
trazado y fundado a partir de ciertas perspectivas teóricas sobre la enseñanza y el aprendizaje.

En esa línea, las exigencias u obligaciones académicas que se les asignan a los estudiantes son hitos que marcan una impronta de las comunicaciones esperadas y por ello, identificar en los materiales y en las distintas secciones de los entornos virtuales cuántas veces, para qué, cuándo y cómo los estudiantes deben obligadamente exponer, enviar y compartir trabajos individuales y/o grupales, son indicadores que nos permiten anticipar y prefigurar cuáles son los eventos de comunicación que se pretende crear y mantener entre profesor y alumnos, y pares entre sí.

Así, en gran parte de la exploración realizada se encontró que las tutorías de los profesores-tutores y las actividades de los alumnos no configuraban obligaciones académicas quedando en el plano de lo opcional, y como tales, no fue posible asignarles el valor de instancias de participación y de intercambio socio-cognitivo ya sea para esclarecer cuestiones conceptuales y metodológicas, verbalizar y exponer los conocimientos, canalizar dudas y preguntas, compartir y re-elaborar significados con otros, dialogar y representar lo aprendido. Asimismo, la escasa presencia de propuestas de trabajo grupal, sea en instancias presenciales o virtuales, es otro indicador del escaso lugar asignado al diálogo, a la escucha, a la negociación de significados, al aprendizaje colaborativo y a la socio-construcción de los conocimientos.

En relación con las tutorías, Duart y Sangrá (2000, p. 38) sostienen que la acción docente en espacios virtuales "tiene como objetivo ofrecer al estudiante herramientas y pistas que le ayuden a desarrollar su propio proceso de aprendizaje, a la vez que atienda sus dudas y necesidades”. En tal sentido, desde los casos analizados se observa que las instancias de consultorías o tutorías si bien tienen la ventaja de estar previstas anticipadamente, en muchos casos resultan ser fechas fijas y rígidas que proponen actividades alejadas de las reales necesidades surgidas durante el cursado. Por otro lado, la falta de registros con datos sobre el desarrollo real de esos espacios tutoriales no permite avanzar sobre los sentidos y características de la comunicación desplegada en esos eventos ni se puede ponderar el valor de la ayuda pedagógica y de la retroalimentación oportuna que se brindan al estudiante. 


\section{Coherencia o grado de concordancia entre el diseño pedagógico- didáctico, la propuesta plasmada en el entorno y el desarrollo efectivo de las interacciones socio-cognitivas}

Esta dimensión resulta relevante en tanto implica develar y caracterizar el tipo de relación que se establece entre el diseño, la propuesta del entorno virtual y el desarrollo efectivo de la red de comunicaciones didácticas. Desde esa perspectiva Duart y Sangrá (2000, p. 173) expresan: "La coherencia de los materiales con la acción docente, con las funcionalidades de su entorno -virtual-y con las relaciones que puedan desencadenarse será fundamental”.

En ese marco, el observatorio permitió caracterizar el nivel de interacciones e intercambios socio-cognitivos entre los actores sobre la base de indicios y evidencias que deja el efectivo desarrollo del circuito de la comunicación en cada caso, donde las heterogeneidades, singularidades y condiciones socioculturales y los perfiles de los sujetos condicionan e inciden en los alcances y matices de resultados y logros. En esa realidad viva e intrincada de cada experiencia es donde se ponen de manifiesto y emergen distintos eventos, complicidades, empatías y sintonías entre profesores y alumnos que imprimen rasgos y aristas singulares a los circuitos prefigurados, con una mayor o menor implicación de los protagonistas en las comunidades discursivas que se generan.

En ese contexto, el análisis conjunto e interrelacionado de los dos momentos de la propuesta formativa, el del diseño académico y el del desarrollo efectivo, es el que posibilita contrastar y dar cuenta del circuito de comunicación didáctica que propone y alcanza cada caso y donde se muestra la intrincada gama de ajustes y desajustes, aceptación de reglas de juego, contradicciones y tensiones de cada situación y de sus actores.

Al analizar la arquitectura pedagógica, didáctica y tecnológica de la pluralidad de casos, se han encontrado evidencias sobre la falta de coherencia y concordancia entre el diseño pedagógico que se sustenta en principios y criterios desde la perspectiva constructivista del aprendizaje que enfatiza el significado de las interacciones socio-cognitivas, los materiales de estudio que no incorporan adecuadamente tales principios y la propuesta académica que se despliega en el entorno virtual y que manifiesta debilidades en torno al número y calidad de espacios grupales y colectivos orientados a la co-construcción de los conocimientos. En tal sentido, se observa que las decisiones e intencionalidades de corte constructivista se diluyen y pierden consistencia cuando se ingresa al itinerario o trayecto pedagógico efectivo que siguen los estudiantes en su formación. 
Estos hallazgos se encuadran en la perspectiva de distintos investigadores que "han encontrado que llevar a los alumnos desde el compartir e intercambiar ideas hasta la construcción de conocimientos es una tarea costosa en la enseñanza online. Los alumnos están dispuestos a compartir ideas pero no a profundizar en el conocimiento a través del debate" (Marcelo García y Perera Rodríguez, 2007, p.392).

Las evidencias de los casos analizados remiten al lugar simbólico y real que se le otorga a la comunicación en los ambientes virtuales, y al sentido y valor que se le atribuye a la tecnología cuyo uso en contextos educativos debería suponer una intervención deliberada e intencional para generar un camino formativo en determinada dirección. En tal sentido Barberá (2008, p. 44) sostiene "es natural pensar que para desarrollar... los procesos de e-learning se precisa una cierta competencia tecnológica, esto es, se necesita la comprensión y la habilidad de conocer dónde y cómo crear una cultura en la cual profesores y alumnos utilicen los ordenadores en una dirección productiva que dé resultados sociales y cognitivos positivos".

Desde un enfoque del aprendizaje de corte constructivista y retomando el pensamiento de Pozo Municio (1998, p. 327) "El aprendizaje como práctica cultural se produce en contextos de interacción, cuyas características afectan seriamente a la eficacia de los resultados obtenidos". Se entiende que es importante ponderar las oportunidades, estrategias y actividades concretas y reales que tributan ese aprender con otros y poner bajo la lupa los trayectos y espacios para el diálogo, la orientación, el apoyo y la evaluación continua, a través de medios y recursos que posibiliten comunicaciones de carácter sincrónico y asincrónico.

\section{REFLEXIONES A MODO DE CIERRE}

Si bien es cierto que las redes virtuales tienen hoy un enorme potencial para generar flujos de comunicación en ambientes de enseñanza y de aprendizaje orientados a la construcción compartida de conocimientos (Borrás, 1997; Ardizzone y Rivoltella, 2004; Barberá, 2008), el análisis de experiencias de educación a distancia en el contexto que se ha descrito, da cuenta, por un lado, de avances cuantitativos representado por el gran número de casos que han adoptado plataformas y, por otro, de ciertas debilidades referidas al escaso aprovechamiento pedagógico de las herramientas tecnológicas para generar comunicaciones de valor cognitivo. 
A partir de las evidencias encontradas tanto en el diseño de las propuestas como en su desarrollo real con el uso de foros, grupos de discusión, chats y blogs, la comunicación orientada al intercambio, diálogo y debate en procura de la construcción compartida de conocimientos, se muestra débil, discontinua y sin intervenciones sistemáticas del profesor que regulen y orienten el trabajo académico colectivo.

Las notas y reflexiones vertidas a partir del observatorio de propuestas educativas virtuales y combinadas, lejos de resistir o desconocer las potencialidades y la eficacia de las herramientas digitales para lograr interacciones socio-cognitivas, intentan destacar una vez más que ellas por sí solas no generan ni son suficientes para una formación sustantiva por cuanto, como instrumentos que son, requieren por un lado, un marco significativo que dé sustento a la propuesta pedagógica y, por otro, un conjunto articulado y consistente de estrategias didácticas que promuevan y apoyen la comunicación tanto sincrónica como asincrónica, y la interacción entre docentes y estudiantes, y alumnos entre sí (Perazzo, 2002; Ardizzone y Rivoltella, 2004; Barberá, 2008).

Asimismo se considera que las tendencias hacia una bimodalidad, con la adopción de la educación a distancia combinada (blended learning) o mixta (García Aretio, 2004; Bartolomé Pina, 2004), constituyen otros modos de resolver la ecuación de cursos y carreras que por su naturaleza, complejidad de competencias a lograr y por las exigencias de actividades de observación y práctica, requieren de una alta supervisión y seguimiento de los aprendizajes, además de espacios de interacción presencial y grupal.

El camino transitado hasta aquí y la práctica siempre alimentada por la pasión hacia el conocimiento, posibilitan sostener que la situación actual resulta desafiante para profundizar líneas de investigación. “Conocer más sobre estos procesos de comunicación y el modo en cómo fluye el discurso significativo en estos entornos, equivale a poder diseñar propuestas innovadoras que amplien las oportunidades de aprendizaje en estos ambientes virtuales” (Marcelo García y Perera Rodríguez, 2007, p.425).

Hoy la realidad se impone como un "ecosistema comunicativo" aunque no faltan voces que interpelan sobre el lugar real de la comunicación, como la del consagrado escritor José Saramago (2002) quien expresa: "Por mucho que las tecnologías de la comunicación se desarrollen y mejoren, siguiendo una auténtica progresión geométrica, la otra comunicación, la comunicación propiamente dicha, la de 
verdad, entre tú y yo, entre nosotros y vosotros, continúa siendo esta confusión cruzada de callejuelas sin salida, engañosamente sembrada de plazas ilusorias, tan engañosa cuando expresa, como cuando quiere ocultar.”

Por ello, dilucidar el lugar y el sentido de la comunicación real en los procesos de educación a distancia sigue siendo un factor clave que posibilita revisar y construir propuestas más enriquecedoras que prefiguren un genuino encuentro y diálogo entre los sujetos. Desde esa vertiente, quizás no sea tan lejano el momento en el que podamos re-encontrarnos y destacar a la educación a distancia por la "comunicación recobrada" -parafraseando nuevamente a Marcel Proust- en tanto intente y logre una comunicación auténtica entre los sujetos.

\section{REFERENCIAS BIBLIOGRÁFICAS}

Abbey, B. (2000). Instructional and cognitive impacts of web-based education. Londres: Idea Group Publishing.

Ardizzone, P.; Rivoltella, P. (2004). Didáctica para e-learning. Málaga: Ediciones Aljibe.

Barberá, E. (2008). Aprender e-learning. Barcelona: Paidós.

Barthes, R. (1993). Fragmentos de un discurso amoroso. México: Siglo XXI Editores.

Bartolomé Pina, A. (2004). Blended learning. Conceptos Básicos. Pixel-Bit, 23. [en línea] Disponible en: http://www.sav. us.es/pixelbit/articulos/n23/PIXEL BIT_23.pdf [consulta 2009, 3 de mayo]

Berger, P.; Luckmann, T. (1972). La construcción social de la realidad. Buenos Aires: Amorrortu editores.

Borrás, I. (1997). Enseñanza y aprendizaje con Internet: una aproximación crítica. Pixel-Bit, 9. [en línea] Disponible en: http://www.sav.us.es/pixelbit/pixelbit/ articulos/n9/ngart/art91.htm [consulta 2009, 11 de marzo]

Breton, P. (2000). La utopía de la comunicación. Buenos Aires: Ediciones Nueva Visión.

Burbules, N. (1999). El diálogo en la enseñanza. Teoría y práctica. Buenos
Aires: Amorrortu.

Burbules, N.; Callister, T. (2001). Riesgos y promesas de las nuevas tecnologías de la información. Barcelona: Granica.

Casas Armengol, M. (1987). Universidad sin clases. Educación a distancia en América Latina. Venezuela: OEA-UNAKapelusz.

Duart, J.; Sangrá, A. (2000) (Comp.). Aprender en la virtualidad. Barcelona: Gedisa.

Freire, P. (1976). Educación y cambio. Buenos Aires: Búsqueda.

Fumero, A.; Roca, G.; Sáez Vacas, F. (2007). Web 2.o. Madrid: Fundación Orange.

Galindo, J. (2004). Hacia una comunicología posible en México. Notas preliminares para un programa de investigación. $X V I$ Encuentro de la Asociación Mexicana de Investigadores de la Comunicación (AMIC). Veracruz.

García Aretio, L. (2004). Blended learning ¿Enseñanza y aprendizaje integrados? Editorial de BENED. [En línea] Disponible en: http://www.uned.es/ catedraUNESCO-ead/editorial/p7-102004.pdf [consulta 2009, 15 de junio]

García Carrasco, J.; Álvarez Cadavid, G. (2008). Reconfiguración como sujetos de comunicación: implicaciones para los 
ambientes virtuales con fines educativos. RUSC, 5 (2) [en línea] Disponible en: http://www.uoc.edu/rusc/5/2/dt/esp/ garcia_alvarez.pdf [consulta 2009, 21 de mayo]

Garrison, D.; Anderson, T. (2005). El elearning en el siglo XXI. Barcelona: Octaedro.

Guardia, L. (2000). El diseño formativo: un nuevo enfoque de diseño pedagógico de los materiales didácticos en soporte digital. En: Duart, J.; Sangrá, A. (Comp.) Aprender en la virtualidad. Barcelona: Gedisa. (171-187).

Gunawardena, C.; Lowe, C.; Anderson, T. (1997). Analysis of a global online debate and the development of an interaction analysis model for examining social construction of knowledge in computer conferencing. Journal Educational Computing Research, 17, 4. (395-429).

Gutiérrez Pérez, F.; Prieto Castillo, D. (1999). La mediación pedagógica. Apuntes para una educación a distancia alternativa. Buenos Aires: Ciccus-La Crujía.

Habermas, J. (1986). Ciencia y técnica como "ideología". Madrid: Tecnos.

Habermas, J. (1988). Teoría de la acción comunicativa. Madrid: Taurus.

Holmberg, B. (1985). Educación a distancia: situación y perspectivas. Buenos Aires: Editorial Kapelusz.

Jorba, J.; Gómez A. I.; Prat, A. (2000). Hablar y escribir para aprender. Uso de la lengua en situaciones de enseñanzaaprendizaje de las áreas curriculares. Madrid: Síntesis.

Kaplún, M. (1983). Hacia nuevas estrategias de comunicación en la educación de adulto. Santiago de Chile: UNESCOOREALC.

Kaye, A.; Rumble, G. (1979). Analysing Distance Learning Sistem. London: Open University.

La Belle, T. (1980). Educación no formal y cambio social en América Latina. México: Editorial Nueva Imagen.
Lauzon, A.; Moore, G. (1989). Fourth Generation Distance Education. The American Journal of Distance Education, 3 (1), (38-49).

Litwin, E.; Libedinsky, M. (1991). La educación a distancia. Deseos y realidades. Buenos Aires: Ediciones OEA.

Marcelo García, C.; Perera Rodríguez, V. (2007). Comunicación y aprendizaje electrónico: la interacción didáctica en los nuevos espacios virtuales de aprendizaje. Revista de Educación, 343, (381-429). [en línea]Disponible en: http://www. revistaeducacion.mec.es/re343/re343_ 17.pdf [consulta 2009, 21 de mayo]

Martín-Barbero, J. (1996). Heredando el futuro. Pensar la educación desde la comunicación. Nómadas, 5, (10-22).

Moore, M. (1975). Cognitive style and telemathic (distance) teaching. ICCE Newsletter, 5 (4).

Niper, S. (1989). Third generation distance learning and computer conferencing. En: Mason y Kaye (Eds.) Mindweave: communication, computers and distance education. Oxford: Pergamon Press, (6373).

O'Sullivan, T.; y otros. (1997). Conceptos clave en comunicación y estudios culturales. Buenos Aires: Amorrortu Editores.

Perazzo, M. (2002). Formación de profesores a distancia: Estudio de un caso de Aprendizaje Colaborativo por Internet. En Moraes (Ed.). Educación a Distancia. Fundamentos y Prácticas. San Pablo: Universidad de Campinas, (137-158).

Perazzo, M.(2008).Larutadelaalfabetización digital en la educación superior: una trama de subjetividades y prácticas. RUSC, 5 (1) [en línea] Disponible en: http://www.uoc.edu/rusc/5/1/dt/esp/ perazzo.pdf [consulta 2009, 9 de mayo]

Pérez Tornero, J. (Comp.) (2000). Comunicacióny educación en la sociedad de la información. Nuevos lenguajes y conciencia crítica. Barcelona: Paidós. 
Pozo Municio, J. (1998). Aprendices y maestros. Madrid: Alianza Editorial.

Salomón, G.; Perkins, D.; Globerson, T. (1992). Coparticipando en el conocimiento: la ampliación de la inteligencia humana con las tecnologías inteligentes. Comunicación, lenguaje y educación, 13, (6-22).

Saramago, J. (2002). El hombre duplicado.
Buenos Aires: Alfaguara.

Taylor, J. (2001). Fifth Generation Distance Education. Higher Education Series, 40.

Watzlawick P.; Beavin Bavelas, J.; Jackson, D. (1991). Teoría de la Comunicación Humana. Interacciones, patologías y paradojas. Barcelona: Editorial Herder.

\section{PERFIL ACADÉMICO Y PROFESIONAL DE LA AUTORA}

Mónica Isabel Perazzo Puncel. Licenciada en Educación Permanente con orientación en Educación a Distancia (UNLu). Magister en Gestión de Proyectos Educativos (CAECE). Profesora Titular de "Comunicación y Educación" de la Universidad Nacional de Lanús. Integrante de la Comisión Federal de Evaluación Permanente de Educación a Distancia. Evaluadora externa de la Comisión Nacional de Evaluación y Acreditación Universitaria (CONEAU). Coordinadora pedagógica del proyecto virtual de Especialización en Educación con orientación en Investigación Educativa (UNLa).

E-mail: mperazzo@me.gov.ar, mperazzo@gmail.com

DIRECCIÓN DE LA AUTORA

Lucio Mansilla $3754 \cdot 6^{\circ} \mathrm{C}$.

(Código postal 1425) Capital Federal

República Argentina

Fecha de recepción del artículo: 30/07/09

Fecha de aceptación del artículo: 06/11/09 